\title{
Prevalencia del consumo de marihuana en estudiantes de una institución de educación superior y los factores asociados a su consumo
}

\author{
Palacios-Arenas Martín Oswaldo*, Terrones-Saldívar Ma. del Carmen**
}

\section{Resumen}

- Introducción: La marihuana es la droga ilícita más consumida - (OMS). En México ha incrementado el consumo de drogas

- ilícitas, siendo el consumo de marihuana en estudiantes uni-

- versitarios de $34.7 \%$ en los hombres y de $19.7 \%$ en las

- mujeres. Objetivo: Determinar la prevalencia del consumo

- de marihuana en universitarios. Metodología: Estudio obser-

- vacional, prospectivo y de corte transversal. Aplicación de la

- encuesta "Cuestionario para estudiantes 20 I 2" del Instituto

- Nacional de Psiquiatría (INP) Dr. Ramón de la Fuente Muñiz

- a estudiantes de la Lic. de Médico Cirujano de la UAA du-

- rante agosto 2016 - enero 2017. Resultados: la prevalencia

- fue de 23.62\% (IC 95\% I 8.9-28.4), en hombres de 28.3\% y

- en mujeres de 19.5\%. La edad de inicio fue de 18.23 1.82

- años, similar en ambos sexos. El 18.8\% es consumidor ex-

- perimental y el $4.8 \%$, regular. La prevalencia fue mayor en

- los semestres $3^{\circ}$ y $4^{\circ}$ y menor en $9^{\circ}$ y $10^{\circ}$. Discusión y

- conclusión: El consumo de marihuana fue mayor al de la

- población general de 18-34 años (23.6\% versus 12.8\%),

- pero menor al de otras series en estudiantes universitarios

- (23.6\% Vs 30\%) p=0.014. Los factores asociados fueron

- problemas físicos o emocionales, baja percepción del riesgo,

- tolerancia social, consumo de sustancias legales y trastorno

- de ansiedad $(p<0.05)$. Se propone aplicar la encuesta en

- otras poblaciones de universitarios para comparar diferen-

- cias en el patrón de consumo y los factores asociados. Se

- sugiere generar estrategias de tamizaje, prevención y mane-

- jo de adicciones en las poblaciones con factores de riesgo.

- LUXMÉDICA AÑO 14, NÚMERO 41, MAYO-AGOSTO 2019, PP 21-30

Palabras clave: marihuana, adicciones, universitarios.

\section{Abstract}

Introduction: According to the World Health Organization (WHO), cannabis is the illicit drug mostly consumed. In Mexico, the use of illicit drugs has increased; cannabis use in university students is $34.7 \%$ in men and $19.7 \%$ in women. Objective: Determine the prevalence of cannabis use in university students. Methods: This is an observational, prospective, and cross-sectional study. The survey "Questionnaire for students 2012" of the INP Dr. Ramón de la Fuente Muñiz was applied to students of the medical degree of the UAA from August 2016 to January 2017. Results: The prevalence was 23.62\% (95\% Cl 1 8.9-28.4), 28.3\% in men and $19.5 \%$ in women. The age of onset was $18.23 \pm 1.82$ years, similar in both sexes; $18.8 \%$ are experimental users, and $4.8 \%$ are regular users. The prevalence was higher in the 3rd and 4th semesters and lower in 9th and 10 th semesters. Discussion and conclusions: The use of cannabis was higher than that of the general population of 1 $8-34$ years (23.6\% vs. 12.8\%) but lower than that of other series in university students (23.6\% vs. 30\%) $p=0.014$. The associated factors were physical or emotional problems, low-risk perception, social tolerance, consumption of legal substances and anxiety disorder $(p<0.05)$. It is proposed to apply the survey in other populations of university students to compare differences in the pattern of consumption and associated factors. It is suggested to generate strategies for screening, prevention, and treatment of addictions in populations with risk factors. LUXMÉDICA AÑO 14, NÚMERO 41, MAYO-AGOSTO 2019, PP 21-30

Keywords: cannabis, addictions, university students 


\section{Introducción}

"Cannabis" se refiere a la planta o productos de ésta con acciones psicoactivas y biológicas tras su consumo. ${ }^{1}$ La marihuana es la droga ilícita más consumida en el mundo con un impacto en 181.1 millones de personas que la utilizan. ${ }^{2}$ La prevalencia estimada en la población general para el continente americano es del $16.5 \%$, siendo mayor en las regiones del norte. ${ }^{3}$ La Encuesta Nacional de Consumo de Drogas, Alcohol y Tabaco (ENCODE) en México ha revelado un incremento en el consumo de drogas ilícitas alguna vez en las personas entre 18-34 años pasando de 5.7\% (2002), 10.6\% (2011) a 14.5\% (2016), situando a la marihuana como la de mayor uso. La región reportada con mayor consumo de marihuana "alguna vez" es la del Nororiente del país con un 12.6\%, pero la región Occidental (se incluye a Aguascalientes) pasó de 9.6\% (2011) a $17 \%$ (2016) con índices ligeramente más altos en zonas urbanas que en las rurales. ${ }^{4-6}$ Para 2014, en los estudiantes de nivel medio o medio-superior, el consumo fue de $17.7 \%$, siendo en los hombres de $28.4 \%$ y en las mujeres de $21.9 \%$. La media nacional del consumo de marihuana es de $10.6 \%$, presentándose de manera similar para el estado de Aguascalientes. ${ }^{7}$ Estudios realizados en universitarios en otros países latinoamericanos indican una prevalencia del $11.58 \%$ siendo menor a la reportada en México, pero con una distribución por sexo similar (mayor en hombres). ${ }^{8}$ En los estudiantes del nivel superior en México, el consumo de marihuana ha sido de $15.1 \%$ a $30 \%$, con predominio en hombres. ${ }^{7,9-11}$

Los efectos de esta droga radican en la afinidad por los receptores cannabinoides CB1 y CB2 en tejido cerebral, intestinal e inmunológico. ${ }^{12}$ Aunque se percibe como una droga poco lesiva presenta repercusiones a corto plazo como la reducción de la memoria, dificultad para el aprendizaje, deterioro de la coordinación motora, alteración del juicio, comportamientos sexuales de riesgo, paranoia y psicosis. Por el consumo prolongado se asocia la adicción, alteración en el desarrollo cerebral, resultado educativo pobre, deterioro cognitivo, reducción del coeficiente intelectual y mayor probabilidad de deserción escolar. ${ }^{1,13}$ Los trastornos por uso de cannabis son definidos por criterios psicológicos, sociales y fisiológicos documentándose efectos adversos, pérdida del control del consumo y manifestación de síntomas del síndrome de abstinencia. ${ }^{14}$

Se han asociado factores individuales con el desarrollo de consumo de marihuana, como: variables genéticas, neuropsicológicas y psiquiátricas. Factores del entorno social: nivel socioeconómico, dificultades para relacionarse, facilidad para adquirir drogas, convivir con consumidores, percepción del peligro, tolerancia social, presión social y prevalencia en su comunidad. Factores del entorno familiar como: disfunción familiar, dinámica familiar, consumo por familiares, distanciamiento de la familia, muerte o enfermedad de alguno de los padres. Factores educativos son: ausentismo, deserción y bajo rendimiento..$^{8,13,15-18}$ Por lo anterior, se identifica que la 


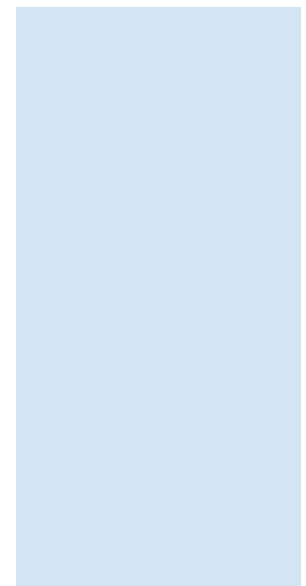

marihuana representa la droga de mayor uso siendo entre el $62 \%$ a $80 \%$ del consumo total de drogas ilegales en México, con un inicio a los 18 años en los hombres y 20 en las mujeres. ${ }^{19}$ Además, tanto centros gubernamentales, no gubernamentales y de integración juvenil para el tratamiento y rehabilitación en adicciones reportan que la droga de impacto más frecuente por la que acudieron a atención fue la marihuana. ${ }^{19-21}$ Aunado a esto, la falta de reportes sobre el consumo de marihuana entre individuos universitarios hace necesaria la presente investigación para contribuir a la comprensión epidemiológica, determinar los factores asociados al consumo y aplicar estrategias para la prevención y atención de un sector de la población potencialmente productivo que tener complicaciones asociadas al uso de marihuana.

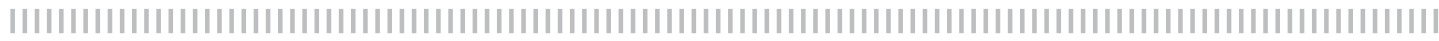

\section{Material y Métodos}

Se realizó un estudio observacional, prospectivo y de corte transversal. Durante el periodo agosto 2016-enero 2017. La muestra se calculó con la fórmula para proporciones en poblaciones finitas con nivel de confianza del $99 \%$, margen de error absoluto del 0.01 y poder de 0.90 , lo cual dio una muestra necesaria de 306. Se hizo muestreo no probabilístico de casos consecutivos. Se incluyeron estudiantes de la carrera de médico cirujano de la UAA, en los semestres de $1^{\circ}$ a $10^{\circ}$ y que aceptaran participar. Se excluyeron a estudiantes de otra carrera, universitarios no inscritos en la UAA o que no aceptaran participar. Se eliminaron las encuestas inconclusas o sin todos los ítems cubiertos. Se usó el "Cuestionario para estudiantes 2012" del INP Dr. Ramón de la Fuente Muñiz, por su uso previo en población de estudiantes

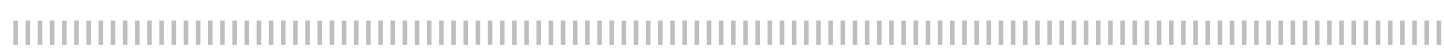

\section{Resultados}

\section{Características sociodemográficas}

Participaron 337 estudiantes, eliminándose 26 encuestas por llenado incorrecto o incompleto. Fueron $164(53.1 \%)$ mujeres y $145(46.9 \%)$ hombres. La edad fue de $20.47+1.73$ años. El $4.2 \%$ indicó vivir solo, $4.2 \%$ solo con otros familiares, mexicanos. Se valoraron las variables sobre el patrón de consumo de marihuana, los aspectos sociodemográficos, consumo de drogas legales e ilegales, problemas relacionados con el consumo de alcohol y otras drogas, conductas antisociales, ámbito personal, ámbito interpersonal, ámbito social, salud mental, rendimiento académico y uso de tiempo libre. Se digitalizó el cuestionario en el sitio web Survio S.R.O ${ }^{\circledR}$. Link de la encuesta: https://www.survio. com/survey/d/X5U9D1Q7W5D104B6Q para que fuese más amigable para el usuario y tuviera mayor confidencialidad. Para el análisis estadístico se usó el programa SPSS 22. Realizando pruebas T de student, $\mathrm{X}^{2}$ y prueba exacta de Fisher con un rango de confianza del $95 \%(\alpha=0.95)$, un margen de error del $5 \%(0.05)$ y una potencia del $90 \%(0.90)$. el $2.6 \%$ solo con amigos, el $0.6 \%$ solo con padre y el $12.3 \%$ solo con madre. El resto presenta una estructura familiar conformada por padre, madre $(71.6 \%)$ y con otros familiares $(4.5 \%)$. El nivel socioeconómico fue muy alto $(39.2 \%)$, alto $(41.4 \%)$, medio-alto $(14.6 \%)$, medio $(4.2 \%)$ y tan sólo $0.6 \%$ medio-bajo. El $20.1 \%$ mencionó presentar algún pro- 
blema físico o emocional, 9.7\% hombres y $10.4 \%$ mujeres.

\section{Prevalencia del consumo de marihuana}

La prevalencia estimada fue de $23.62 \%$ (IC 95\% 18.9-28.4) para el consumo alguna vez en su vida. Fue más frecuente en los hombres (28.3\% [IC 95\% 20.9-35.6]) que en las mujeres (19.5\% [IC 95\% 13.425.6]), pero no significativo $\left(X^{2}=3.276\right.$ $p=0.07)$. La edad de inicio del consumo fue de 18.23+1.82 años, similar en ambos sexos. De los consumidores alguna vez, 46.6\% (IC 95\% 35.1-58) la ha utilizado en el último año, mientras que el 1.9\% (IC 95\% 0.4-3.5) en el último mes.
De éstos, el 1.0\% de 1-5 días, el $0.6 \%$ de $6-19$ días y el $0.3 \%>20$ días. El $18.8 \%$ (IC 95\% 14.4-23.1) es consumidor experimental, mientras que el $4.8 \%$ (IC 95\% 2.5-7.9) es consumidor regular, siendo el 7.6\% (IC 95\% 3.3-11.9) de los hombres y el $2.4 \%$ (IC 95\% 0.1-4.8) de las mujeres. En ambos sexos, el cigarro o puro (64\%) y la vía fumada (83.6\%) fueron las formas más frecuentes de consumo, el resto de la distribución por formas de administración se presenta en la Tabla No.1. El motivo del consumo por primera vez más frecuente fue "para identificar si le gustaba" $(54.8 \%)$ y se proporcionó inicialmente por un amigo o conocido (83.6\%).

\section{Tabla 2}

Productos de marihuana y vías de administración entre los consumidores alguna vez. La vía inyectada presentó una frecuencia de 0 , por lo que no se incluyó.

\begin{tabular}{|c|c|c|c|c|c|c|}
\hline \multicolumn{7}{|c|}{ FORMAS Y VÍAS DE CONSUMO DE MARIHUANA } \\
\hline & $\begin{array}{l}\text { Hombre } \\
(n=41)\end{array}$ & IC $95 \%$ & $\begin{array}{l}\text { Mujer } \\
(n=32)\end{array}$ & IC 95\% & $\begin{array}{l}\text { Total } \\
(n=73)\end{array}$ & IC 95\% \\
\hline \multicolumn{7}{|c|}{ Producto } \\
\hline Aceites o alimentos & $31.7 \%$ & $17.5-46$ & $15.6 \%$ & $3-28.2$ & $24.7 \%$ & $14.8-34.5$ \\
\hline Ungüentos & $14.6 \%$ & $3.8-25.5$ & $6.3 \%$ & $-2.1-14.6$ & $11 \%$ & 3.8-18.1 \\
\hline Pipas sólidas & $56.6 \%$ & $40.9-71.3$ & $37.5 \%$ & $20.7-54.3$ & $47.9 \%$ & $36.5-59.4$ \\
\hline Cigarros o puros & $70.7 \%$ & $56.8-84.7$ & $56.3 \%$ & $39.1-73.4$ & $64.4 \%$ & $53.4-754$ \\
\hline Pipas de agua & $24.4 \%$ & $11.2-37.5$ & $9.4 \%$ & $-0.7-19.5$ & $17.8 \%$ & $9-26.6$ \\
\hline \multicolumn{7}{|c|}{ Vías de administración } \\
\hline Fumada & $90.2 \%$ & $76.8-97.3$ & $75 \%$ & $60-90$ & $83.6 \%$ & $75.1-92.1$ \\
\hline Inhalada & $2.4 \%$ & $-2.3-7.2$ & $6.3 \%$ & $-2.1-14.6$ & $4.1 \%$ & $-0.4-8.7$ \\
\hline Bebida & $34.1 \%$ & $19.6-48.7$ & $21.9 \%$ & $7.6-36.2$ & $28.8 \%$ & $60.8-81.6$ \\
\hline Untada & $12.2 \%$ & $2.2-22.2$ & $9.4 \%$ & $-0.7-19.5$ & $11 \%$ & $3.8-18.1$ \\
\hline
\end{tabular}


En la figura No.1 se muestra que la mayor prevalencia encontrada fue en los semestres $3^{\circ}$ y $4^{\circ}$ para el consumo "alguna vez", en los últimos 12 meses y en el último mes $(28.4 \%, 14.9 \%$ y $3 \%$, respec- tivamente). Los semestres con menor prevalencia fueron los de $9^{\circ}$ y $10^{\circ}$ para "alguna vez" y en el "último mes" (18.2\% y $9.1 \%)$.

\section{Prevalencia del consumo de marihuana por grado}

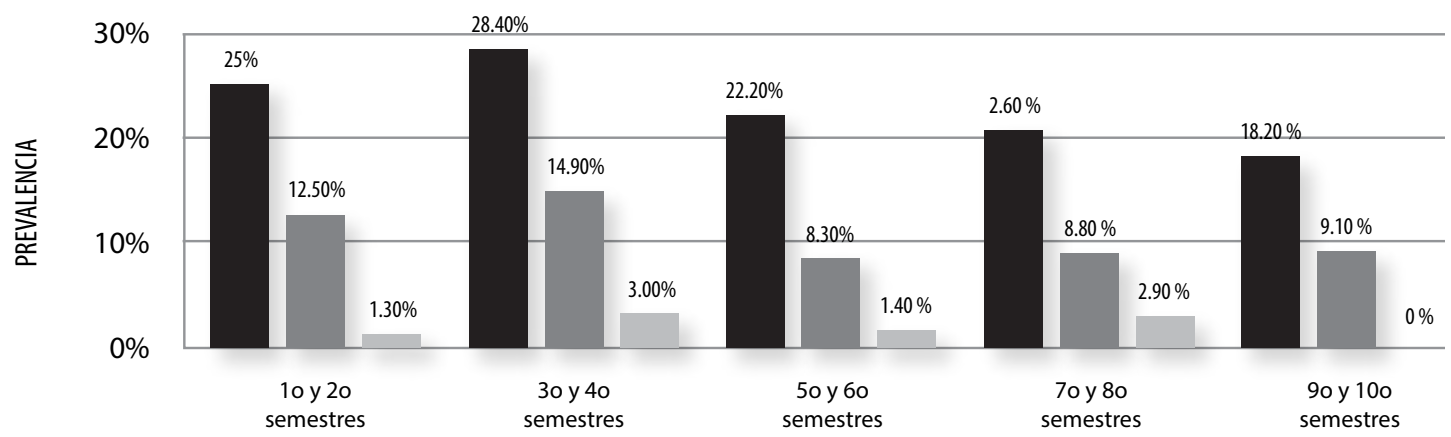

Semestre que cursa

\footnotetext{
Alguna vez en la vida

En los últimos 12 meses
}

En el último mes

Figura No. 1. Prevalencia del consumo de marihuana por semestres. Se realizó la $X^{2}$ pero no se identificó asociación estadísticamente significativa para ninguno de los patrones de consumo y el semestre que cursa.

\section{Factores asociados}

Vivir con amigos presenta asociación con una mayor prevalencia de consumo respecto a los que habitan con padre, madre u otros familiares $(37.5 \%$ vs $10.29 \%)$ (P. Fisher $\mathrm{p}=0.041)$. También se identificó que el consumo de alcohol o tabaco alguna vez en su vida se asoció con el consumo "alguna vez de marihuana" $\left(\left[\mathrm{X}^{2}=\right.\right.$ $8.684 p=0.003] ;\left[X^{2}=50.604 p<0.0001\right]$, respectivamente). La edad temprana de consumo de alcohol (15.75+1.96 años) y tabaco (16.35+2.03 años) se asoció con el consumo de marihuana "alguna vez" ([T de $S t=3.91 p<0.0001]$; [T de $s t=2.95$ $\mathrm{p}=0.004]$, correspondientemente). Además, los que han fumado tabaco en los últimos 12 meses tienen una prevalencia notoriamente mayor (39.86\%) de consumo de marihuana de los que no $(9.65 \%)$; $\left(X^{2}=38.887 p<0.0001\right)$. Finalmente en los estudiantes que refirieron acudir "siempre" a fiestas o a eventos con amigos donde consumen alcohol tienen mayor prevalencia de uso de marihuana alguna vez y en los últimos 12 meses que aquellos que indican hacer dichas actividades nunca o sólo algunas veces; $64.29 \%$ vs $12 \%$ y $29.63 \%$, para asistencia con amigos $\left(X^{2} p<0.0001\right)$, $52.63 \%$ vs 13.48 y $25.37 \%$, para asistencia a fiestas $\left(X^{2} p<0.0001\right)$. El resto de los factores con asociación estadísticamente significativa se muestra en las tablas No. 3, No. 4 y No. 5 . 


\section{Tabla 3}

Se muestran los factores familiares asociados con el consumo de marihuana. * = no hay asociación estadísticamente significativa.

\begin{tabular}{|c|c|c|c|c|c|}
\hline \multicolumn{6}{|c|}{ Factores familiares asociados al consumo de marihuana } \\
\hline \multirow[t]{3}{*}{ Factor } & \multicolumn{5}{|c|}{$n=309$} \\
\hline & \multicolumn{2}{|c|}{ Usó alguna vez } & \multicolumn{3}{|c|}{ Usó últimos 12 meses } \\
\hline & & $\%$ & Prueba & $\%$ & Prueba \\
\hline \multirow[t]{3}{*}{ Convivencia con madre } & Casi no convive/nada & 31.91 & $X^{2}=7.790 p=0.020$ & 10.63 & $X^{2}=9.988 p=0.040$ \\
\hline & $1-2$ horas/día & 32.39 & & 10.63 & \\
\hline & $>3$ Horas/día & 18.32 & & 9.94 & \\
\hline \multirow[t]{2}{*}{ Convivencia con padre } & $>5$ Horas/día & 9.09 & $X^{2}=4.325 p=0.038$ & ${ }^{*}$ & ${ }^{*}$ \\
\hline & $<5$ Horas/día & 25.36 & & & \\
\hline \multirow[t]{3}{*}{ \# personas que fuman en el hogar } & 0 & 20.7 & P. Fisher $=8.223 p=0.025$ & 9.25 & P. Fisher $=12.234 p=0.039$ \\
\hline & $1-2$ & 29.49 & & 15.39 & \\
\hline & $>3$ & 75 & & 33.33 & \\
\hline \multirow[t]{2}{*}{ Hermano/s consumen drogas } & No & 20.72 & $X^{2}=11.628 p=0.001$ & 8.72 & P. Fisher $=13.030 p=0.001$ \\
\hline & Sí & 47.06 & & 29.41 & \\
\hline \multirow[t]{2}{*}{ Familiares con problemas por alcohol } & No & 14.66 & $x^{2}=22.278 p<0.0001$ & 7.85 & $x^{2}=23.173 p<0.000$ \\
\hline & Sí & 38.14 & & 16.10 & \\
\hline
\end{tabular}

\section{Tabla 4}

Se muestran los factores socio-ambientales estadísticamente asociados con el consumo de marihuana.

\begin{tabular}{|c|c|c|c|c|c|}
\hline \multicolumn{6}{|c|}{ Factores socio-ambientales asociados al consumo de marihuana } \\
\hline \multirow[t]{3}{*}{ Factor } & \multicolumn{5}{|c|}{$n=309$} \\
\hline & \multicolumn{3}{|c|}{ Usó alguna vez } & \multicolumn{2}{|c|}{ Usó últimos 12 meses } \\
\hline & $\%$ & & Prueba estadística & $\%$ & Prueba estadística \\
\hline \multirow[t]{2}{*}{ Le han ofrecido drogas regaladas } & No & 7.47 & $X^{2}=57.594 p<0.0001$ & 8.57 & $X^{2}=57.843 p<0.0001$ \\
\hline & Sí & 44.44 & & 34.48 & \\
\hline \multirow[t]{2}{*}{ Le han ofrecido drogas vendidas } & No & 21.43 & $x^{2}=7.974 p=0.005$ & 8.57 & P. Fisher $=13.557 p=0.001$ \\
\hline & Sí & 44.83 & & 34.48 & \\
\hline \multirow[t]{4}{*}{ Piensas que conseguir drogas es: } & Imposible & 4.25 & $X^{2}=16.339 p=0.003$ & 0 & $X^{2}=26.779 p=0.001$ \\
\hline & Muy difícil & 20.97 & & 1.61 & \\
\hline & Fácil & 29.6 & & 16.3 & \\
\hline & Muy fácil & 40.74 & & 22.22 & \\
\hline \multirow[t]{2}{*}{ Mejor amigo consume drogas } & No & 15.09 & $X^{2}=27.235 p<0.0001$ & 3.3 & $X^{2}=43.205 p<0.0001$ \\
\hline & Sí & 42.27 & & 27.84 & \\
\hline \multirow[t]{3}{*}{$\begin{array}{l}\text { Percepción de peligro para el } \\
\text { consumo de marihuana }\end{array}$} & No peligroso & 50.8 & $x^{2}=38.547 p<0.0001$ & 33.33 & $x^{2}=51.188 p<0.0001$ \\
\hline & Peligroso & 22.38 & & 7.7 & \\
\hline & Muy peligroso & 8.73 & & 1.94 & \\
\hline \multirow[t]{3}{*}{ Amigos que consumen drogas } & Ninguno & 14.64 & P. Fisher $=14.844 p<0.0001$ & 2.55 & P. Fisher $=27.951 p<0.0001$ \\
\hline & Algunos & 32.65 & & 19.05 & \\
\hline & La mayoría & 40 & & 40 & \\
\hline \multirow[t]{3}{*}{$\begin{array}{l}\text { ¿Cómo verían tus amigos que tú } \\
\text { consumieras drogas? }\end{array}$} & Mal & 11.56 & P. Fisher $=47.038 p<0.0001$ & 3.52 & P. Fisher $=49.586 p<0.0001$ \\
\hline & Ni bien, ni mal & 45.28 & & 23.58 & \\
\hline & Bien & 50 & & 50 & \\
\hline
\end{tabular}




\section{Tabla 5}

Se muestran los factores individuales asociados con el consumo de marihuana. * = no hay asociación estadísticamente significativa. ** Sólo valorable para consumo de marihuana alguna vez. AUDIT27: Cuestionario de Identificación de los Trastornos debidos al Consumo de Alcohol de la OMS. Zona I: abstinencia o bajo riesgo. Zona II: sobre bajo riesgo, consumo de riesgo. Zona III: consumo de riesgo o perjudicial. Zona IV: dependencia.

\begin{tabular}{|c|c|c|c|c|c|}
\hline \multicolumn{6}{|c|}{ Factores individuales asociados al consumo de marihuana } \\
\hline \multirow[t]{3}{*}{ Factor } & \multicolumn{5}{|c|}{$n=309$} \\
\hline & & \multicolumn{2}{|c|}{ Usó alguna vez } & \multicolumn{2}{|c|}{ Usó últimos 12 meses } \\
\hline & & $\%$ & Prueba & $\%$ & Prueba \\
\hline \multirow[t]{2}{*}{ Problema físico o emocional } & No & 21.1 & $X^{2}=4.513 p=0.034$ & 10.9 & $X^{2}=7.167 p=0.028$ \\
\hline & Sí & 33.9 & & 11.3 & \\
\hline \multirow[t]{4}{*}{ Fumar diariamente } & Nunca fumar & 9.64 & $x^{2}=60.288 p<0.0001$ & 3.61 & $X^{2}=65.933 p<0.0001$ \\
\hline & Actualmente no fuma & 25 & & 8.33 & \\
\hline & Frecuentemente & 47.83 & & 26.09 & \\
\hline & Todos los días & 68 & & 40 & \\
\hline \multirow[t]{3}{*}{ \# de copas por ocasión } & $1-2$ copas & 13.68 & P. Fisher $=36.385 p<0.0001$ & 5.13 & $x^{2}=39.304 p<0.0001$ \\
\hline & 5-6 copas & 41.03 & & 23.08 & \\
\hline & $>10$ copas & 50 & & 33.33 & \\
\hline \multirow[t]{4}{*}{ Zona de AUDIT } & Zona 1 & 22.41 & P. Fisher $=24.297 p<0.0001$ & 8.71 & P. Fisher $=31.962 p<0.0001$ \\
\hline & Zona 2 & 55.56 & & 40.74 & \\
\hline & Zona 3 & 33.33 & & 16.67 & \\
\hline & Zona 4 & 50 & & 50 & \\
\hline \multirow[t]{2}{*}{$\begin{array}{l}\text { Trastorno de ansiedad } \\
\text { Hamilton }\end{array}$} & No & 21.14 & $X^{2}=4.134 p=0.042$ & ** & * \\
\hline & Sí & 33.33 & & & \\
\hline \multirow[t]{2}{*}{$\begin{array}{l}\text { Inicio de vida sexual } \\
\text { activa (IVSA) }\end{array}$} & No & 11.04 & $X^{2}=26.951 p<0.0001$ & 4.55 & $x^{2}=39.124 p<0.0001$ \\
\hline & Sí & 36.13 & & 17.42 & \\
\hline \multirow[t]{3}{*}{$\begin{array}{l}\text { Tipo de relación con } \\
\text { persona de IVSA }\end{array}$} & Amistad & 48.72 & $X^{2}=11.203 p=0.011$ & 30.77 & $x^{2}=14.406 p=0.025$ \\
\hline & Casual & 83.33 & & 33.32 & \\
\hline & Noviazgo & 29.36 & & 11.92 & \\
\hline \multirow[t]{2}{*}{$\begin{array}{l}\text { Relaciones sexuales y } \\
\text { consumo de alcohol }\end{array}$} & No & 27.36 & $X^{2}=11.177 p=0.001$ & 10.38 & $X^{2}=14.119 p=0.001$ \\
\hline & Sí & 35.1 & & 32.65 & \\
\hline
\end{tabular}

\section{Discusión}

En la serie actual la prevalencia del consumo de marihuana fue mayor a lo reportado en la ENCODE 2017 para la población general de entre 18 a 34 años $(23.6 \%$ vs $12.8 \%)$, siendo mayor tanto en hombres (28.3\% vs $20 \%$ ) como en mujeres $(19.5 \%$ vs $6.1 \%) .4,5,19$ También fue mayor a lo reportado en series de universitarios en el estado de Sinaloa para el consumo alguna vez $(15.1 \%$ vs $23.6 \%$ ) y en el último año $(5.4 \%$ vs $11 \%){ }^{10}$ Sin embargo, menor a los reportes de la ciudad de México (30\% vs $23.6 \%$ ), estadísticamente significativa $\mathrm{p}=0.014 .{ }^{9}$ La distribución por sexo resultó mayor en los hombres que en las mujeres, concordando con publicaciones previas en población general y universitarios., ${ }^{75,19} \mathrm{La}$ 
edad de inicio fue similar a lo reportado en la ENCODET 2017.4,5,19 Por frecuencia de utilización, el grupo de consumidores regulares es más reducido que lo estimado entre los estudiantes del Estado de México (4.8\% vs $5.9 \%$ ). Además, al igual que lo que se ha documentado, los productos de marihuana que más se utilizaron fueron los cigarros o puros y la vía de administración más prevalente fue la fumada, siendo similar en ambos sexos y grupos de edad. ${ }^{1,21}$

\section{Factores asociados}

En el ámbito individual, los que destacan son la presencia de problema físico o emocional, baja percepción del riesgo del consumo, consumo temprano de drogas legales, consumo problemático de las mismas, actividades en las que usa el tiempo libre y trastornos de ansiedad $(p<0.05)$, afín a lo descrito por González y Ortiz., donde mencionan que el patrón de consumo de sustancias puede presentarse como modelo ascendente iniciando de forma temprana con drogas legales para posteriormente usar las ilegales. ${ }^{22-24}$ En el estudio de Blanco y el de Phillip, se ha asociado el consumo de marihuana con trastornos psiquiátricos hallándose la relación que existe entre el consumo y trastornos depresivos, de ansiedad y de adicción al alcohol o al tabaco. 25,26 Ello es concordante con lo observado en el presente estudio, ya que hubo asociación entre la presencia de ansiedad y el consumo de marihuana alguna vez $\left(X^{2}=4.134\right.$ $p=0.042)$; el abuso o dependencia de alcohol ([50\% para AUDIT zona 4 y $33.33 \%$ para zona 3 vs $22.41 \%$ para Zona 1] [P. Fisher $=24.297 \mathrm{p}<0.0001]) ;$ la adicción al tabaco $(68 \%$ para fumar todos los días vs $9.64 \%$ para nunca fumar $X^{2}=60.288$ $p<0.0001)$. Sin embargo, en la población estudiada no se identificó asociación con la presencia de trastorno depresivo, ideación o intento suicida y la poca autoestima con el consumo de marihuana. ( $p>0.05)$ Además, el patrón de actividad sexual se asoció con el uso de marihuana $\left(X^{2}=11.177\right.$ $p=0.001)$ concordando con lo publicado por Moure y cols., reportando la relación de patrones sexuales de riesgo y el consumo de cannabis. ${ }^{27}$

En el grupo de componentes familiares, es importante mencionar que la estructura familiar no presentó relevancia estadísticamente significativa con mayor consumo de marihuana y fue más relevante el tiempo de convivencia con la madre, con el padre o vivir con la madre, difiriendo de series previas donde mencionan la importancia de la estructura familiar o la funcionalidad de la misma. ${ }^{22}$ Además, presentar familiares con problemas asociados al alcohol, consumo de drogas ilegales o tabaquismo por familiares $(p<0.05)$ fue semejante a lo descrito en otras poblaciones de universitarios. ${ }^{23,24,26}$

Para el grupo de factores sociales la percepción de pocos riesgos negativos asociados al consumo de marihuana, amistades y actividades recreativas donde el consumo de drogas legales o ilegales sea habitual fue análogo a lo descrito en reportes previos donde explican que estos factores se afianzan con expectativas de bienestar, tranquilidad y evitación de problemas, así como la necesidad de ser un sujeto perteneciente a la comunidad que lo circunscribe y se suma a la facilidad de acceso o disponibilidad de la marihuana u otras drogas en el entorno que se desarrolla el adolescente o joven..$^{22-24}$ 


\section{Conclusiones}

La prevalencia del consumo de marihuana en la población universitaria evaluada es menor al compararla con otras series publicadas en México. Dichas diferencias quizá radiquen en las características sociodemográficas que presenta el grupo evaluado, ya que la mayoría tiene un nivel socioeconómico elevado. Otro factor que puede influir sobre el consumo de marihuana es que la población encuestada estudia una carrera afín al cuidado de la salud, por lo cual la percepción del riesgo asociado al consumo de sustancias psicoactivas puede ser mayor en contraste con otras poblaciones. Se recomienda hacer estudios complementarios para determinar los factores implicados en el desarrollo del consumo de marihuana en otras poblaciones universitarias, con lo cual se puedan generar modelos más completos para realizar intervenciones de tamizaje, prevención y difusión sobre la problemática del consumo de marihuana y sus repercusiones en la salud, el desarrollo personal y el desempeño profesional. Lo anterior, fundamentándose en la importancia que tienen las universidades como formadores de individuos con las herramientas necesarias para afrontar problemas y tomar decisiones, así como la promoción del autocuidado y prácticas sociales benéficas.

\section{Bibliografía}

1.- Volkow D. Baler R. Compton W. Weiss S. Adverse Health Effects of Marijuana Use. The New England Journal of Medicine. 2014; 370: 2219-2227.

2.- Organización Mundial de la Salud. The health and social effects of nonmedical cannabis use. 2016. Consultado en:

http://www.who.int/substance_abuse/publications/msb cannabis report.pdf?ua $=1$. (Fecha de consulta: $\overline{2}$ 5-08-2016).

3.- Oliva N. Villatoro J. Mujica R. Fregoso D. Bustos M. Medina M. Panorama Actual del Consumo de Sustancias en Estudiantes de la Ciudad de México. Instituto Nacional de Psiquiatría Ramón de la Fuente Muñiz. 2015. Administración Federal de Servicios Educativos en el Distrito Federal. Instituto para la Atención y Prevención de las Adicciones; México, D.F.

4.- INPRFM, INSP, SSA. Encuesta Nacional de Adicciones (ENA) 2011: Reporte de Drogas. Instituto Nacional de Psiquiatría Ramón de la Fuente Muñiz; Instituto Nacional de Salud Pública; Secretaría de Salud. 2012. México DF, México: INPRFM. Consultado en: http://www.conadic.salud.gob.mx/pdfs/ena_2011_ drogas_ilicitas_pdf. (Fecha: 3-09-2016)

5.- Villatoro V. Resendiz E. Mujica A. Et al. Encuesta Nacional de Consumo de Drogas, Alcohol y tabaco 2016-2017: reporte de Drogas (ENCODET). Instituto Nacional de Psiquiatría Ramón de la fuente Muñiz; Instituto Nacional de Salud Pública; Secretaría de Salud. 2017. Ciudad de México. Consultado en: https://www.gob.mx/salud\%7Cconadic/ acciones-y-programas/encuesta-nacional-deconsumo-de-drogas-alcohol-y-tabaco-encodat-2016-2017-136758. (Fecha: 22-3-29)

6.- Consejo Estatal de Población. Programa Estatal de Población: 2011-2016. 2014. Coordinación Estatal de Planeación de y Proyecto del Gobierno del Estado de Aguascalientes

7.- Villatoro V. Oliva R. Fregoso I. Et al. Encuesta Nacional de Consumo de Drogas en Estudiantes 2014: Reporte de Drogas. Instituto Nacional de Psiquiatría Ramón de la Fuente Muñiz; Comisión Nacional Contra las Adicciones. 2015. Secretaría de Salud. México DF, México: INPRFM.

8.- Rojas V. Rodríguez C. Zereceda G. Otiniano C. Abuso de drogas en adolescentes y jóvenes y vulnerabilidad familiar. Oficina de las Naciones Unidas Contra la Droga y el Delito. 2013. Biblioteca Nacional del Perú Nº 2013-19282. Perú.

9.- Comisión Nacional Contra las Adicciones. Pautas para la Prevención y la Atención de las Adicciones en Universidades, Institutos y Escuelas de Educación Media Superior y Superior en México. 2012. Secretaria de Salud. México, D.F. Consultado en: http:// www.conadic.salud.gob.mx/pdfs/pautas_prevencion.pdf (Fecha de consulta: 03-09-2016)

10. Guzmán F. Llamas L. Rodríguez L. Alonso M. Norma subjetiva, intención y consumo de marihuana en jóvenes universitarios de México. Revista de ciencia y enfermería. 2012. 18; 57-66.

11. Villatoro J. Mendoza M. Moreno M. Oliva N. Fregoso D. Bustos M. Fleiz C. Mujica R. López M. MedinaMora M. Tendencias del uso de drogas en la Ciudad de México: Encuesta de estudiantes, octubre 2012. 
Salud Mental. 2014; 37: 423-435.

12. Bostwick J. Medicinal Use of Marijuana. The New England Journal of Medicine. 2013; 368: 866-968.

13. Curran V. Freeman T. Mokrysz C. Lewis D. Morgan C. Parsons L. Keep off the grass? Cannabis, cognition and addiction: Review. Nature Reviews Neuroscience. 2016; 17: 293-306.

14. APA. Diagnostic and statistical manual of mental Disorders, fifth edition. Arlington (VA): American Psychiatric Association. 2013.

15. Consejo Nacional Contra las Adicciones. Encuesta del Estado de México sobre Consumo de Alcohol, Tabaco y Drogas en Estudiantes. Instituto Mexiquense contra las Adicciones; Secretaria de Salud; Instituto Nacional de Psiquiatría Ramón de la Fuente Muñiz. 2009 Consultado en: http://www.uade. inpsiquiatria.edu. $\mathrm{mx} /$ pagina_contenidos/investigaciones/LIBROS\% 20EDOMEX/Encuesta \% 20del\% 20 Estado\% 20de\% 20Mexico, \% 20sobre \% 20consumo\%20de\%20drogas\%20en \% 20estudiantes, \% 20 2009.pdf. (Fecha de consulta: 04-09-2016).

16. Bustos E. Algunos factores relacionados con las adicciones. Revista de Especialidades Médico-Quirúrgicas. 2012; 17: 69-70.

17. Aguilar E. Valdez G. López M. Gallegos A. Camacho R. Mendoza M. Problematic consumption of drugs and risk or protection factors in a reclusion center of the city of Mexico. International Journal of Psychological Research. 2013;6:78-83.

18. Guimaraes G. Mendoza M. López M. Et al. (2014). Prevalencia y factores asociados al consumo de tabaco, alcohol y drogas en una muestra poblacional de adultos mayores del Distrito Federal. Revista de Salud Mental. 2014; 37: 15-25.

19. Villatoro J. Medina-Mora M. Fleiz C. Et al. El consumo de drogas en México: Resultados de la Encuesta Nacional de Adicciones, 2011. Revista de Salud Mental. 2012; 35: 447-457.

20. Villatoro J. Moreno M. Oliva N. Et al. Consumo de Alcohol, Tabaco y otras Drogas en la Ciudad de México: Medición 2012. Instituto Nacional de Psi- quiatría Ramón de la Fuente Muñiz. Instituto para la Atención y la Prevención de las Adicciones, Administración Federal de los Servicios Educativos para el Distrito Federal, México, D.F. 2013.

21. Oficina de las Naciones Unidas Contra la Droga y e Delito (UNODC). Informe Mundial Sobre las Drogas. Publicación de las Naciones Unidas. 2015; S.15.XI.6.

22. González K. Londoño C. Factores personales, sociales, ambientales y culturales de riesgo de consumo de marihuana en adolescentes. Psicología y Salud. 2017; 27: 141-153.

23. Ortiz M. Trejo M. Blázuez M. Pavón P. Barreto $P$. Pérez $M$. Factores psicosociales asociados al consumo de drogas en estudiantes de dos universidades de américa Latina. Rev. Salud Pública. 2018; 22: 41-52.

24. Fleming C. Guttmannova K. Cambron C. Rhew I. Oesterle $\mathrm{S}$. Examination of the Divergence in trends for Adolescent Marihuana Use and Marijuana-Specific Risk Factors un Washington State. Journal Adolescent Health. 2017; 59: 269-275.

25. Blanco C. Hasin D. Wall M. Et al. Cannabis Use and Risk of Psychiatric Disorders Prospective Evidence From a US National Longitudinal Study. JAMA Psychiatry. 2016; 73: 388-95.

26. Phillips K. Phillips M. Duck K. Factors Associated With Marijuana use anda Problems Among College Students in Colorado. Substance use y misuse. 2017. 0: 1-7.

27. Moure L. Doallob S. Salvadores J. Corral M. Cadaveira F. Caamano F. Consumo intensivo de alcohol y cannabis, y prácticas sexuales de riesgo en estudiantes universitarios. Gaceta Sanitaria. 2016; 30: 438443.

28. Organización Mundial de la Salud. AUDIT: Cuestionario de Identificación de los trastornos debidos al consume de alcohol, pautas para su utilización en atención primaria. Consultado en: http://www.who. int/substance abuse/activities/en/AUDITmanualspanish.pdf. 2001. Última vista. (Fecha de consulta: 20-11-2016) 